\title{
Die Gestaltung Forschenden Lernens mit digitalen Medien
}

\section{Carola Schirmer und Victoria Marín}

Mit diesem Beitrag möchten wir zur Nutzung digitaler Medien in Prozessen des Forschenden Lernens anregen, indem wir Beispiele und Kriterien für den Einsatz digitaler Medien aufzeigen. Wie in anderen Anwendungsbereichen orientiert dieser sich auch beim Forschen und Lernen an den Aktivitäten, die zur Erreichung bestimmter Ziele stattfinden. Es stellt sich daher nicht die Frage, ob die Nutzung digitaler Technologien im Forschenden Lernen an sich sinnvoll ist, sondern welche Aktivitäten im Forschenden Lernen stattfinden und wie diese jeweils mit digitalen Anwendungen unterstützt werden können.

Angemessen eingesetzt können digitale Medien die Arbeitsschritte im Forschenden Lernen und damit die Erreichung didaktischer Ziele unterstützen. Die Orientierung an diesen Zielen bedeutet, sie zu analysieren, bevor digitale Unterstützungssysteme ausgewählt, angepasst und genutzt werden. Gelingt diese Gestaltung des Einsatzes der Technologie, so kann die Umsetzung von Forschendem Lernen in der Lehre dadurch gestärkt werden.

Zunächst werden kurz Merkmale und theoretische Grundlagen des Forschenden Lernens dargestellt. Im Anschluss werden Kriterien beschrieben, die sich daraus für die Nutzung digitaler Technologien ergeben. Exemplarisch werden einige Anwendungen für ausgewählte Aktivitäten vorgestellt. 


\section{$1 \quad$ Lerntheoretische Aspekte und Kriterien für die Gestaltung Forschenden Lernens mit digitalen Medien}

Für den Einsatz von technischen Systemen gilt grundsätzlich, dass ihre Entwicklung oder Auswahl den mit ihnen zu bewältigenden Aufgaben und zu erreichenden Zielen entsprechen muss. Für die digitale Gestaltung des Lehrens und Lernens kommt dies in der Rede vom „Primat der Pädagogik“ zum Ausdruck (z. B. BMBF, 2016; Kultusministerkonferenz, 2016). Pädagogische und didaktische Ziele stehen an erster Stelle. Die Nutzung digitaler Medien soll Kriterien und Anforderungen folgen, die aus diesen Zielen abgeleitet werden. Hier skizzieren wir daher lerntheoretische Aspekte des Forschenden Lernens und arbeiten Kriterien für die technische Unterstützung heraus. Darauf aufbauend werden Beispiele für digitale Medien dargestellt, die für Aktivitäten des Forschenden Lernens angemessen sind.

\subsection{Forschendes Lernen: konstruktivistisch, situiert und konnektivistisch}

Lernprozesse als Forschendes Lernen zu gestalten lässt sich lerntheoretisch mit Konstruktivismus, Konnektivismus und situiertem Lernen begründen. Neues Wissen eignen sich Lernende demnach aktiv an, indem sie neue Informationen in Verbindung mit ihrem Vorwissen zu sinnhaften neuen Konstrukten verknüpfen (Siebert, 1998). Studierende lernen so durch selbstständiges Forschen zu Fragestellungen, die sie selbst erarbeiten. Prozesse des Lernens finden „situiert“ statt, eingebunden in Handlungskontexte einer Community of Practice (Lavé \& Wenger, 1991). Dies können zum Beispiel die Angehörigen einer Profession sein. Wissen und Bedeutungen werden im Handeln und in der Interaktion dieser Community erzeugt, bestätigt und weiterentwickelt. Aus der Perspektive des Konnektivismus (Siemens, 2005) bedeutet Lernen, neue Knotenpunkte durch Verknüpfung vorhandenen Wissens zu schaffen.

Forschendes Lernen und Forschung finden häufig in Form von Projekt- und Gruppenarbeit statt. Neues Wissen wird gemeinsam im Austausch unterschiedlicher Perspektiven und arbeitsteilig erarbeitet. Daher sind Schwerpunkte der Nutzung digitaler Medien im Forschenden Lernen die Kollaboration, die Kooperation und die Kommunikation unter Studierenden sowie zwischen Lehrenden und Studierenden.

Mit der theoretischen Konzeption von Lernprozessen (nicht nur) im Forschenden Lernen als konstruktivistisch, situiert und konnektivistisch sowie als kollaborative 
oder arbeitsteilige Prozesse bieten sich digitale Medien zur Unterstützung des vernetzten Arbeitens und interaktiver Wissenskonstruktion an.

\subsection{Kriterien der Auswahl digitaler Tools für das Forschende Lernen}

Digitale Tools für das Forschende Lernen unterstützen Lernprozesse, die konstruktivistisch, situiert und konnektivistisch gestaltet werden, ebenso das selbstbestimmte Vorgehen und kollaborative Arbeiten der Studierenden.

Die Selbstbestimmheit des Forschenden Lernens wird ermöglicht, wenn Studierende die Auswahl und Nutzung digitaler Tools entsprechend ihrem Vorgehen mitbestimmen können. Die digitalen Systeme sollten nicht mit einem unveränderbaren Rollenkonzept (z. B. Lehrende /Studierende) arbeiten, sondern eine Zusammenarbeit und Kommunikation ohne Hierarchie ermöglichen, sowohl unter Studierenden als auch zwischen Studierenden und Lehrenden. Die Studierenden können dabei über die öffentliche, eingeschränkte oder private Sichtbarkeit ihrer Produkte mitentscheiden.

Kollaboratives Arbeiten und Projektarbeit wird durch digitale Medien ermöglicht, welche die Kommunikation und Zusammenarbeit der Lernenden unterstützen. Koordinative Funktionen erlauben dabei eine Mitgestaltung der Studierenden bei Terminplanung und Fristen, Aufgabenverteilung und Zusammenarbeit sowie einen kollaborativen Zugriff auf Materialien und Kommunikation.

Gemäß dem Ansatz des situierten Lernens sollten Studierende eingebettet in der „Community of Practice“ ihres Fachgebiets lernen. Es sollten Technologien zum Einsatz kommen, die in der Forschung oder Berufspraxis des Faches tatsächlich verwendet werden, sowie Technologien, die eine Einbindung und Vernetzung in der Fachcommunity ermöglichen.

Konstruktivistische Aspekte des Lernens können unterstützt werden, indem Studierende Forschungsfragen, ihr Vorgehen, Ergebnisse und neu Gelerntes mithilfe digitaler Medien schriftlich oder visuell darstellen. Sie konstruieren dabei ihre eigene Perspektive und bauen neues Wissen gemäß ihrem eigenen Zugang zu einem Thema auf.

Nach dem theoretischen Ansatz des Konnektivismus bedeutet Lernen die Nutzung und neue Verknüpfung von Wissen, das bei Menschen, aber auch in Netzwerkknoten (Organisationen, Technologien) vorhanden ist. Studierende sollten daher lernen, Wissen in digitalen Netzwerken zu finden, zu strukturieren und zu verknüpfen. 


\section{Die Nutzung digitaler Tools in den verschiedenen Phasen des Forschenden Lernens}

Aktivitäten des Forschendes Lernen lassen sich den Phasen eines zyklischen Forschungsprozesses zuordnen (Huber, 2014, S. 23; Bundesassistentenkonferenz, 1970; Sonntag et al., 2016, S. 14):

1. Wahrnehmen eines Ausgangsproblems (Hinführung)

2. Erarbeiten einer Fragestellung

3. Erarbeiten von Informationen und theoretischen Zugängen

4. Auswahl und Aneignung von Methoden

5. Entwicklung eines Forschungsdesigns

6. Durchführung der Forschung

7. Erarbeitung und Präsentation der Ergebnisse

8. Reflexion des gesamten Prozesses

Einige Aktivitäten sind fachübergreifend gleich, andere fachspezifisch und je nach Forschungsansatz unterschiedlich. Die Bestimmung geeigneter digitaler Medien setzt eine Analyse des Forschungsverlaufs eines Faches bzw. einzelner Lehrveranstaltungen voraus (Marín \& Schirmer, 2018). Wir betrachten exemplarisch einige solcher Aktivitäten und beschreiben, welche Art digitaler Medien sich zu ihrer Unterstützung eignet. Dies schließt nicht aus, dass andere als die beispielhaft genannten Tools ebenfalls gut geeignet sein können.

\subsection{Phasenübergreifende Aktivitäten}

Unabhängig von einzelnen Phasen gibt es übergreifende Aktivitäten im Forschenden Lernen, bei denen die Nutzung digitaler Tools Vorteile bietet. Diese können darin bestehen, dass Kommunikation und Information orts- und zeitunabhängig für alle Beteiligten verfügbar sind und zudem übersichtlich strukturiert sowie auf Dauer gesichert werden können. Zur Koordination können Projektmanagementtools genutzt werden. Für die Kommunikation in Teams können E-Mail, Messenger oder Plattformen für Gruppenarbeit eingesetzt werden. Texte oder Präsentationen können gemeinsam online geschrieben, kommentiert und überarbeitet werden. Je nach fachlichen Anforderungen stehen unterschiedliche Systeme für Textverarbeitung oder Präsentation zur Verfügung (z. B. Office-Systeme, LaTex- oder XML-basierte Systeme, Blog, Etherpad, Wiki). Die Nutzung solcher Systeme entspricht den oben genannten Kriterien der Zusammenarbeit und der selbstbestimmten Organisation 
und bietet Möglichkeiten für konnektivistisch vernetztes und konstruktivistisches Vorgehen bei der Erarbeitung neuen Wissens.

\subsection{Beispiele für Aktivitäten in den Forschungsphasen und Unterstützung durch digitale Medien}

Beim „Erarbeiten einer Fragestellung“ (Phase 2) können Studierende gemeinsam eine Themenlandkarte erstellen (Sonntag et al., S. 89). Hierfür eignen sich Online-Tools für MindMaps oder ConceptMaps, welche die gemeinsame und gleichberechtigte Bearbeitung ermöglichen (z. B. Mindomo oder Cmaptools). Ebenfalls können Pinnwand-Tools zur gemeinsamen Strukturierung genutzt werden. Dabei erarbeiten sich die Studierenden ihre eigenen Zugänge zu einem Thema (Abb. 1).

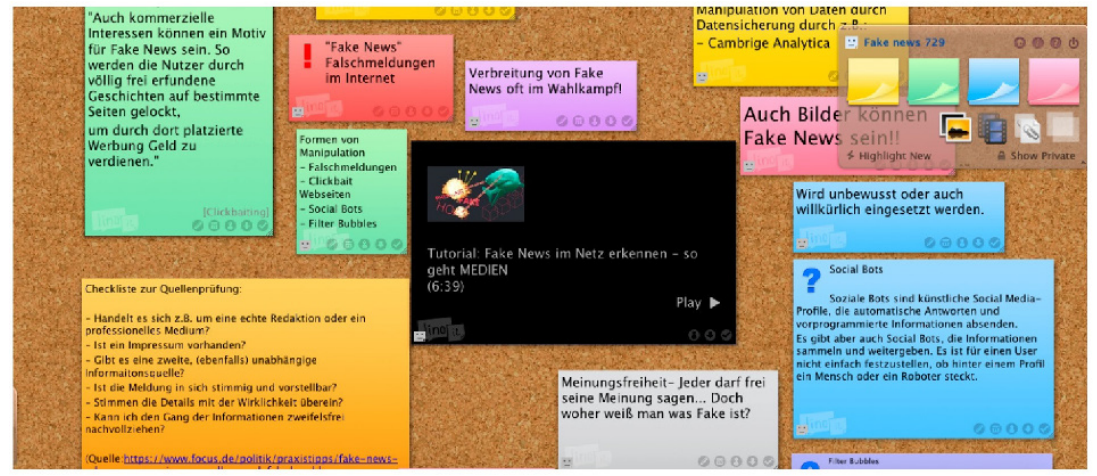

Abb. 1 Das Thema „Fake News“ mit der Online-Pinnwand Linoit dargestellt (Linoit, http://linoit.com/)

Für das „Erarbeiten von Informationen und theoretischen Zugängen“ (Phase 3) recherchieren Studierende wissenschaftliche Publikationen über digitale Rechercheinstrumente wie Kataloge und Fachdatenbanken, aber auch in sozialen Medien wie Blogs, Microblogging- und Community-Plattformen (z. B. Researchgate). Zur Bearbeitung und Strukturierung werden digitale Literaturverwaltungssysteme genutzt, von denen manche eine Zusammenarbeit ermöglichen (z. B. RefWorks oder Zotero). Auch die gemeinsame Bearbeitung von Literatur kann durch webbasierte PDF-Reader, die gemeinsame Kommentare vorsehen, unterstützt werden. Beim 
Lesen können Markierungen und Kommentare gesetzt werden, die von anderen einsehbar sind und wiederum kommentiert werden können (z. B. Hypothesis, NowComment oder Mendeley) (Abb. 2). Studierende lernen dabei - situiert - die Arbeits- und Kommunikationsweisen ihres Fachgebiets kennen.

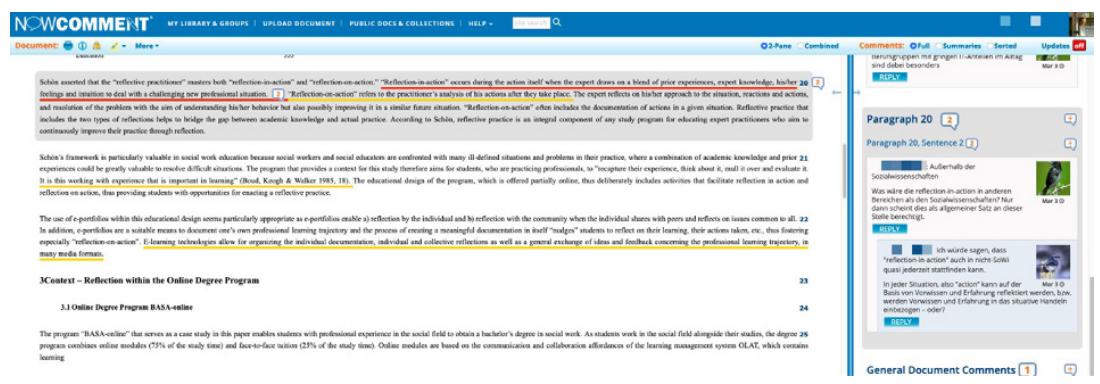

Abb. 2 Diskussion der Lesenden zu einem Dokument in NowComment, http:// nowcomment.com

Die „Durchführung der Forschung“ (Phase 6) unterscheidet sich je nach Fachgebiet. In den Sozialwissenschaften können für Online-Umfragen digitale Tools eingesetzt werden (z.B. SurveyMonkey oder LimeSurvey). Zur Auswertung und Modellierung werden Softwarepakete genutzt (z. B. SPSS, SAS oder R). Qualitative Forschung durch Interviews und Textanalysen geschieht ebenfalls digital gestützt (z. B. MaxQDA). Insbesondere in der Phase der Forschungsdurchführung erlernen Studierende Methoden ihres Fachgebiets und wachsen in die Praxis der Fachcommunity herein. Das Erlernen des Umgangs mit den entsprechenden Technologien entspricht daher dem Kriterium des situierten Lernens.

Für die „Erarbeitung und Präsentation von Ergebnissen“ (Phase 7) können digitale Medien wie Online-Zeitschriften oder Blogs genutzt werden. Texte können bei Gruppenarbeiten mithilfe von digitalen Tools gemeinsam verfasst oder für Peer-Feedback und Feedback von Lehrenden genutzt werden. Auch Ergebnisdarstellungen mit Videos, Bildern oder in anderen Formaten lassen sich digital erstellen und präsentieren.

Die Reflexion des gesamten Lern- und Forschungsprozesses kann begleitend oder nach Abschluss (Phase 8) durch ein E-Portfolio unterstützt werden, in dem die Lernenden ihre Arbeit und ihren Lernprozess reflektieren (Abb. 3). 


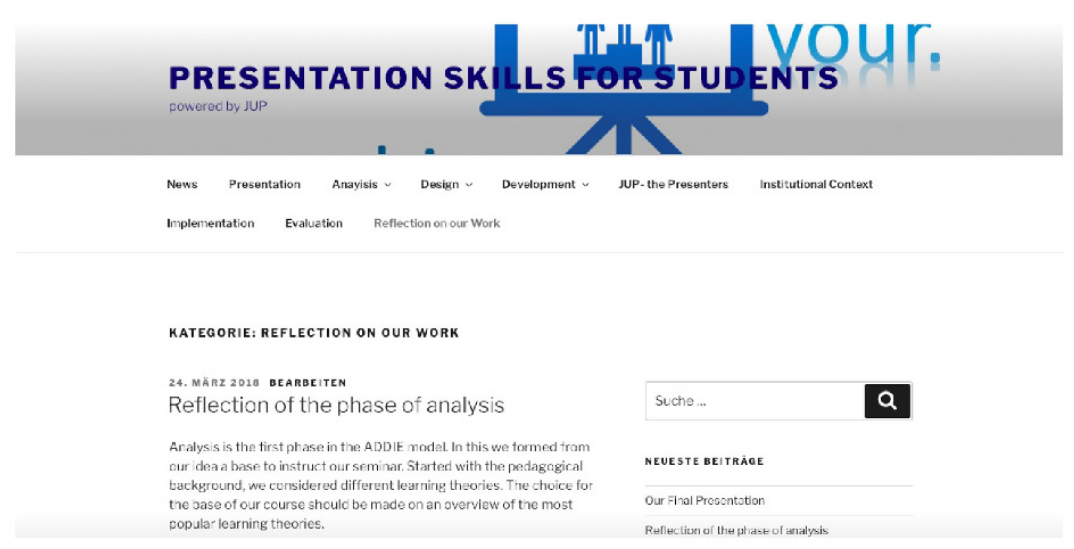

Abb. 3 WordPress Blog als E-Portfolio für die Reflexion der Arbeit von Studierenden

\section{Diskussion von Hindernissen und Rahmenbedingungen beim Einsatz digitaler Medien}

Lernprozesse im Format des Forschenden Lernens beinhalten, dass Studierende möglichst selbstgesteuert vorgehen. Da heißt, sie planen auch den Ablauf der Forschung mit und bearbeiten alle anfallenden Arbeitsschritte. Für Lehrende ist es eine zentrale Herausforderung, den Studierenden dabei Entscheidungsspielräume zu geben und zugleich sicherzustellen, dass sie wichtige Lernprozesse durchlaufen (Wiemer, 2017). Digitale Medien können Lehrende bei dieser Aufgabe unterstützen, indem sie zum Beispiel mit Studierenden unkompliziert kommunizieren, den Arbeitsstand einsehen und auch direktes Feedback geben können. Um durch digitale Medien im Forschenden Lernen solche positiven Effekte zu erzielen, sind jedoch entsprechende Rahmenbedingungen zu schaffen.

Sowohl Lehrende als auch Studierende benötigen Einarbeitungszeit und müssen die Handhabung der jeweiligen Tools erlernen. Die praktische Erfahrung zeigt, dass die Einführung von Tools in der Präsenzzeit von Lehrveranstaltungen vorteilhaft ist, damit Studierende Einstiegshürden überwinden und diese tatsächlich nutzen.

Neben der technischen Einarbeitung ist die gemeinsame Arbeit mit den digitalen Tools in der Veranstaltung selbst auch sinnvoll, damit Lehrende und Studierende eine neue, zeitgemäße Lernkultur entwickeln können. Zu dieser gehört unter anderem, zu lernen und auszuhandeln, wie mit den Tools kollaborativ gearbeitet 
werden soll, Vertrauen aufzubauen, um Arbeitsprozesse offen und transparent zu gestalten, sowie zu lernen, wie Feedback gegeben und mit Feedback umgegangen werden kann. Erst im Rahmen einer neuen Lernkultur - im Sinne von Teilhabe an einer Community of Practice - können sich die Potenziale der digitalen Vernetzung entfalten (Bower, 2017).

Ein wichtiger Aspekt bei der Nutzung digitaler Systeme ist der Schutz persönlicher Daten. Grundsätzlich ist davon auszugehen, dass die Nutzung von Software und Cloud-Diensten, die von der Hochschule angeboten und auf eigenen Servern gehostet werden, rechtlich unbedenklich ist. Neben Lernmanagementsystemen bieten viele Hochschulen inzwischen auch andere digitale Medien an, zum Beispiel Blogs. Bei anderen Anbietern, welche die Nutzerdaten auf Servern außerhalb der Hochschule speichern, müssen Lehrende sich zuvor über eine rechtskonforme Nutzung informieren.

Zusammenfassend lässt sich festhalten, dass digitale Medien viele Möglichkeiten bieten, Forschendes Lernen umzusetzen und erfolgreich zu gestalten. Wichtig ist dabei, dass lerntheoretische Grundlagen des situierten Lernens, des Konstruktivismus und Konnektivismus beachtet werden und immer die didaktischen Ziele im Fokus der Wahl digitaler Medien stehen.

\section{Literatur}

Bower, M. (2017). Design of Technology-enhanced Learning: Integrating Research and Practice. Bingley: Emerald Publishing Limited.

Bundesassistentenkonferenz (BAK) (1970). Forschendes Lernen - Wissenschaftliches Prüfen (Schriften der BAK, 5. Neudruck). Bielefeld: UVW.

Bundesministerium für Bildung und Forschung (BMBF) (2016). Bildungsoffensive für die digitale Wissensgesellschaft. Berlin: BMBF. Verfügbar unter https://www.bmbf.de/pub/ Bildungsoffensive_fuer_die_digitale_Wissensgesellschaft.pdf [15.08.2018].

Huber, L. (2014). Forschungsbasiertes, Forschungsorientiertes, Forschendes Lernen: Alles dasselbe? Ein Plädoyer für eine Verständigung über Begriffe und Unterscheidungen im Feld forschungsnahen Lehrens und Lernens. Das Hochschulwesen, 62(1 \& 2) S.22-29.

Kultusministerkonferenz (KMK) (2016). Strategie der Kultusministerkonferenz: „Bildung in der digitalen Welt" (Beschluss der Kultusministerkonferenz). Berlin: KMK. Verfügbar unter https://www.kmk.org/fileadmin/Dateien/pdf/PresseUndAktuelles/2016/ Bildung_digitale_Welt_Webversion.pdf [15.08.2018].

Lavé, J. \& Wenger, E. (1991). Situated Learning: Legitimate peripheral participation. Cambridge: Cambridge University Press.

Marín, V. I. \& Schirmer, C. (2018): Design of a teacher-training workshop to support research-based learning processes with digital media. In N. Neuber, W. Paravicini \& 
M. Stein (Hrsg.), Forschendes Lernen: The Wider View. Eine Tagung des Zentrums für Lehrerbildung der Westfälischen Wilhelms-Universität Münster vom 25. bis 27.09.2017 (Schriften zur Allgemeinen Hochschuldidaktik, Band 3, S.99-110). Münster: WTM.

Siebert, H. (Hrsg.) (1998). Konstruktivismus: Konsequenzen für Bildungsmanagement und Seminargestaltung (Materialien für Erwachsenenbildung, 14). Frankfurt: Deutsches Institut für Erwachsenenbildung.

Siemens, G. (2005). Connectivism: A Learning Theory for the Digital Age. International Journal of Instructional Technology and Distance Learning, 2(1). Verfügbar unter https:// pdfs.semanticscholar.org/a25f/84bc55488d01bd5f5acac4eed0c7d8f4597c.pdf [24.08.2019]. Sonntag, M., Rueß, J., Ebert, C., Friederici, K. \& Deicke, W. (Hrsg.) (2016). Forschendes Lernen im Seminar: Ein Leitfaden für Lehrende. Berlin: Humboldt-Universität zu Berlin. Wiemer, M. (2017). Forschend lernen - Selbstlernen: Selbstlernprozesse und Selbstlernfähigkeiten im Forschenden Lernen. In H. A. Mieg \& J. Lehmann (Hrsg.), Forschendes Lernen: Wie die Lehre in Universität und Fachhochschule erneuert werden kann. (S.47-55). Frankfurt: Campus.

\section{Zu den Autorinnen}

Carola Schirmer ist Mitarbeiterin für Hochschuldidaktik und e-Didaktik sowie für die Digitalisierung der Lehre im Referat Studium und Lehre an der Carl von Ossietzky Universität Oldenburg. E-Mail: carola.schirmer@uni-oldenburg.de

Dr. Victoria Marín ist Wissenschaftliche Mitarbeiterin im Bereich „Wissenstransfer und Lernen mit neuen Technologien" und in der Hochschuldidaktik (e-Didaktik) an der Carl von Ossietzky Universität Oldenburg. E-Mail: victoria.marin@ uni-oldenburg.de

Open Access Dieses Kapitel wird unter der Creative Commons Namensnennung 4.0 International Lizenz (http://creativecommons.org/licenses/by/4.0/deed.de) veröffentlicht, welche die Nutzung, Vervielfältigung, Bearbeitung, Verbreitung und Wiedergabe in jeglichem Medium und Format erlaubt, sofern Sie den/die ursprünglichen Autor(en) und die Quelle ordnungsgemäß nennen, einen Link zur Creative Commons Lizenz beifügen und angeben, ob Änderungen vorgenommen wurden.

Die in diesem Kapitel enthaltenen Bilder und sonstiges Drittmaterial unterliegen ebenfalls der genannten Creative Commons Lizenz, sofern sich aus der Abbildungslegende nichts anderes ergibt. Sofern das betreffende Material nicht unter der genannten Creative Commons Lizenz steht und die betreffende Handlung nicht nach gesetzlichen Vorschriften erlaubt ist, ist für die oben aufgeführten Weiterverwendungen des Materials die Einwilligung des jeweiligen Rechteinhabers einzuholen.

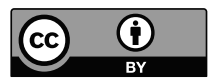

